\title{
Updated Prediction of Aggregators and Assay \\ Interfering Substructures in Food Compounds
}

Andrés Sánchez-Ruiz \& Gonzalo Colmenarejo*

Biostatistics and Bioinformatics Unit

\author{
IMDEA Food \\ CEI UAM+CSIC \\ E28049 Madrid, Spain
}

${ }^{*}$ Corresponding Author

e-mail: gonzalo.colmenarejo@imdea.org 


\section{ABSTRACT}

1 Positive outcomes in biochemical and biological assays of food compounds may appear

2 due to the well-described capacity of some compounds to form colloidal aggregates that

3 adsorb proteins, resulting in their denaturation and loss of function. This phenomenon

4 can lead to wrongly ascribing mechanisms of biological action for these compounds

5 (false positives), as the effect is non-specific and promiscuous. Similar false positives can

6 show up due to chemical (photo)reactivity, redox cycling, metal chelation, interferences

7 with the assay technology, membrane disruption, etc., which are more frequently

8 observed when the tested molecule has some definite interfering substructures.

9 Although discarding false positives can be achieved experimentally, it would be very

10 useful to have in advance a prognostic value for possible aggregation and/or

11 interference, based only in the chemical structure of the compound tested, in order to

12 be aware of possible issues, help in prioritization of compounds to test, design of appropriate assays, etc. Previously, we applied cheminformatic tools derived from the drug discovery field to identify putative aggregators and interfering substructures in a database of food compounds, the FooDB, comprising 26457 molecules at that time. Here we provide an updated account of that analysis based on a current, muchexpanded version of the FooDB, comprising a total of 70855 compounds. In addition, we also apply a novel machine learning model (the SCAM Detective) to predict aggregators with $46 \%-53 \%$ increased accuracies over previous models. In this way, we expect to provide the researchers in the mode of action of food compounds with a much

21 improved, robust, and widened set of putative aggregators and interfering substructures of food compounds. 
24 KEYWORDS: Food compounds, aggregators, interference filters, PAINS, assay

25 interference, promiscuous compounds, cheminformatics, SCAM Detective 
27 There is currently a great research effort in the identification of the biological mechanisms of action of food compounds from a molecular point of view, in order to understand the beneficial or harmful effects of foods on human health, as well as finding novel nutraceuticals and scaffolds for drug design. ${ }^{1-9}$ For that aim, biochemical and/or biological (cellular) assays directed towards different biological targets (typically proteins) are being conducted, so that specific macromolecule-food compound interactions can be identified. However, these assays are subject to compound-related artifacts. For example, compound aggregation is a well-described phenomenon that yields artifacts in biochemical and biological assays. ${ }^{10-14}$ Some compounds, due to low water solubility, when tested at concentrations above a critical aggregation concentration $(\mathrm{CAC}),{ }^{15}$ form colloidal aggregates that adsorb biomacromolecules nonspecifically and alter their activities, in most cases inhibiting them through denaturation, ${ }^{16}$ although in some cases activating them. ${ }^{17}$ This effect can translate into misled interpretations of the biological mechanism of action of compounds, as it is wrongly ascribed to the target used in the assay while the aggregation is nonspecific. Aggregation is very dependent on the assay conditions $(\mathrm{pH}$, buffer composition, testing concentration) and structure of the compound, rather than on the assay technology and target, and can be alleviated to some extent by the addition of nonionic detergents in the assay medium.

An alternative source of false positives in assays is the presence of substructures that make the molecule interact promiscuously with many targets, through mechanisms like (photo)chemical reactivity, large hydrophobicity, redox cycling, metal chelation, etc.; or 
that provide it with some interfering properties with the assay technology (absorption/emission at reading wavelengths, membrane disruption, singlet-oxygen quenching or production, etc.). ${ }^{18-26}$

There is a soaring concern for the presence of increasing numbers of false positives in the scientific literature and the derived databases of bioactivities due to aggregation and/or interfering substructures, as it could severely hurt knowledge extraction, decision making, and lead to the waste of resources and time. ${ }^{27,28}$ As a matter of fact, the American Chemical Society has provided specific guidelines ${ }^{28}$ to follow when submitting manuscripts reporting biological or biochemical activities of compounds with putative aggregation and/or interfering substructures, such as performing additional orthogonal assays (with the same target but different technology) and/or counterassays (with different target but the same technology), adding non-ionic detergents in the assay buffer, and reviewing reported activities for the same molecules in the literature.

In a previous work, ${ }^{29}$ we performed an systematic analysis of a large database of food compounds, the FooDB, ${ }^{30}$ aiming at identifying there both putative aggregators and interfering substructures. This effort would be useful for the scientific community aiming at deciphering the biological mechanisms of action of food compounds, as it would allow to point out possible issues with reported activities, guide in the prioritization of compounds to test, and help in the assay technology selection and design. We used for the analysis well-established and publicly available cheminformatic tools, ${ }^{12,19,20,22}$ derived from the statistical and machine learning analysis of a large number of structure-activity datasets from both the high-throughput screening and 
medicinal chemistry fields: to identify putative aggregators, we employed the Aggregator Advisor model, ${ }^{12}$ while to find putative interfering substructures, we used three standard libraries of substructural filters derived through data mining efforts: the Pan-Assay INterference compoundS ("PAINS") set, ${ }^{22}$ that of former-GlaxoWellcome (here called "Glaxo"), ${ }^{19}$ and another from Pfizer ("LINT"). ${ }^{20}$

Since 2020, the FooDB size has increased considerably, from about 26000 compounds at the time when the previous paper was published, to about 71000 to date. This suggested the need for an update of this analysis, as the number of compounds in the current version more than duplicates that of the previous one, leaving a lot of molecules without analysis for possible aggregation and/or interfering behavior. In addition, we took the opportunity to use more advanced approaches to predict aggregation. The Aggregator Advisor uses a simple and conservative approach for prediction, as it relies on a database of 12000 known aggregators, and only marks a new compound as aggregator if its Tanimoto similarity to one or more know aggregators is $>0.85$ and its $\log P>3$. Thus, this approach is restricted to a chemical space very close to the known aggregators, not being able to extrapolate to molecules outside this narrow space. It basically suggests putative aggregators with confidence, but potentially misses the vast majority of the chemical space, and contains no information about non-aggregators. On the contrary, a recent model based on machine learning, the so-called SCAM Detective, ${ }^{31}$ is based on balanced datasets comprising tens of thousands of compounds for both the aggregator and non-aggregator classes, in two assays, each repeated with and without detergent, and at different concentrations of compound (here SCAM stands for "small, colloidally aggregating molecules"). The use of a much larger and balanced 
95 dataset, together with a Random Forest as predictive model, as well as Extended-

96 Connectivity Fingerprints of diameter 6 (ECFP6) to represent molecular structures,

97 allows to increase the sensitivity and specificity of the predictions, resulting in

98 improvements of total accuracy from $46 \%$ to $53 \%$ over previous models (e.g. the

99 Aggregator Advisor), and to make it applicable to the whole chemical space. In addition,

100 the SCAM Detective allows to generate probability maps for aggregation, that highlight

101 regions in the molecule with high and low propensity for aggregation and help in the 102 interpretation of the predictions in molecular terms.

103 Thus, in this work we attempt to provide an updated and more robust analysis of the 104 putative aggregators and interference substructures in the current FooDB, comprising 105 near 71000 molecules. We expect this work will be valuable for the experimentalist 106 testing food compounds in different biological targets, in order to reduce the presence 107 of false positive reports in the literature for these molecules. In turn, this will help 108 globally to gain a better understanding of their true biological mechanisms of action and 109 structure-activity relationships. 
111 All the analyses were performed in Python 3.9 and with the RDKit cheminformatic 112 toolkit, ${ }^{32}$ version 2021.03.3. The FooDB, ${ }^{30}$ comprising a total of 70855 molecules 113 (including all detected and quantified, detected but not quantified, expected but not 114 quantified, and predicted) were kindly provided by Dr. Wishart group in SDF format. 115 Structures were checked in a first step, and then standardized and solvent- and counterion-stripped (in the case complex molecules containing counterions and/or solvent molecules), to yield the corresponding parent compound, by using the ChEMBL Structure Pipeline. ${ }^{33}$ A few compounds could not be processed by RDKit. Some compounds raising the Pipeline's exclusion flag (i.e. with transition metals, or $>7$ of boron atoms, that cannot be properly standardized) or with penalty scores $>5$ (due to severe structural inconsistencies) were discarded (molecules with penalty score $=5$ were kept as these corresponded to stereo mismatches between InChi vs RDKit vs Mol representations of the molecules, with no impact in the analysis of aggregators or interference substructures). After that, duplicates were removed based on the InChiKey, resulting in a total of 69502 unique molecules. The application of this recently described structure normalization procedure changed slightly the statistics for the molecules analyzed in our previous work and reported therein, ${ }^{29}$ that were now reanalyzed as they were a subset of the new FooDB, but we think this provides a more robust analysis of aggregators and interference substructures, without changing the former conclusions. 
132 status as in our previous work, and following normalization procedure as with the

133 FooDB. The resulting number of unique molecules was 2154.

134 For the SCAM Detective calculations to predict aggregators, ${ }^{31}$ the code provided by the 135 authors was locally installed and adapted for batch calculations. For comparison 136 purposes with our previous work, the aggregators were also predicted through the 137 Aggregator Advisor method, ${ }^{12}$ implemented in a locally programmed script with RDKit 138 as the Aggregator Advisor does not provide batch processing functionality. In this script, 139 a molecule was assigned a "known" status if its Tanimoto similarity with a molecule in 140 the list of 12642 Aggregator Advisor molecules was 1, a status of "probable" if its largest 141 Tanimoto similarity with any molecule in the list of aggregators was $\geq 0.85$ and its $\log P$ $142>3$, and an status of "none" for the rest of the molecules.

143 To test differences in distributions of numeric variables in different groups, the nonparametric Kruskal-Wallis one-way analysis was used, followed by Conover's post-hoc test with p-values correction using Holm's approach. To test differences in proportions 146 for pairs of samples, Z-test for proportions were used. 


\section{Physicochemical and structural analysis of the molecules in the new FooDB}

Prior to conducting our analysis of aggregators and interfering substructures, it seemed necessary a characterization of the molecules incorporated in the new release of the FooDB, in order to see the extent of overlap with the chemical space of the previous FooDB molecules. A very large subset of the additional molecules in the new FooDB corresponds to acylglycerols (in what follows abbreviated AG), namely compounds with a glycerol backbone and with at least one fatty acid esterified to it, although other types of molecules (non-acylglycerols or NAG) are present. Figure 1 displays different in-silico calculated physicochemical and structural properties for four groups of molecules, specifically the molecules in the previous FooDB (corresponding to the "FD(OLD)" label in the abscissa), new non-acylglycerol molecules in FooDB (likewise, "FD(NEW/NAG)"), new acylglycerol molecules in FooDB ("FD(NEW/AG)"), and DrugBank (“DB") molecules. The descriptors calculated were: TPSA (topological polar surface area, TPSA), IogP (LOGP), number of rotatable bonds (RB), number of hydrogen bond donors (HBD), number of hydrogen bond acceptors (HBA), molecular weight (MW), QED (Quantitative Estimation of Drug-likeness, $\mathrm{QED}^{35}$ ), number of rings (NRING), and fraction of sp3 carbons (FSP3).

We can see in Figure 1 that the dispersion of distributions is quite different for NAG and AG molecules in the new FooDB molecules. The main descriptive statistics and test for differences (omnibus and post-hoc) in the distributions of all the four groups of molecules can be obtained in Supporting Information (Tables S1 and S2). While the NAG tend to have a widening of their distributions as compared to the old FooDB, the AG 
171 have usually very narrow distributions, with TPSA, HBD, HBA and NRING having a null

172 inter-quartile range, which is expected given the high structural redundancy within this

173 group as they are all acyl esters of glycerol. Both NAG and AG display a trend towards 174 more and larger LOGP, RB, HBA, MW and FSP3. This is especially clear for the AG as 175 regarding LOGP, RB and FSP3, and expected given their highly aliphatic structure. On the 176 other hand, both NAG and AG display a reduction in QED and NRING, again not 177 unexpected for the AG molecules.

178 As regarding TPSA, NAG show a marked increase compared to the old FooDB, while AG show an slight decrease of it; in the case of the HBD, the AG molecules have no one, while NAG show a clear trend towards higher values.

On the other hand, Figure 2 displays the distributions of the 10 most frequent BemisMurcko ${ }^{36,37}$ scaffolds for FDB(OLD), FDB(NEW/NAG), and DB. AG molecules are not shown as any of them have scaffold (they are linear, branched molecules, and therefore have no Bemis-Murcko scaffolds, which are ring-based). Again, we observe quite different distributions for these sets, in spite the benzene ring being the most frequent scaffold in all of them. The percentage of molecules without scaffold is lowest in the drugs, but highest in the NAG (after AG, which are 100\%). Following the benzene scaffold, the drugs show a variety of typical drug scaffolds; in decreasing order, two steroid scaffolds, pyridine, diphenylmethane, another steroid, etc. In turn, the old FooDB molecules have in common the presence of steroid scaffold, but the second most frequent one is cyclohexane, followed by tetrahydropyran, etc., while the NAG compounds display completely different scaffolds: e.g. dual aliphatic esters ending in furan rings, cyclopentamine, imidazole, etc. 
a different region of the chemical space (including physicochemical properties and structures), more separated to that of the drugs, as compared to the previous release of FooDB. This observation stresses the need for an updated analysis of aggregators and interference substructures in the FooDB.

\section{Aggregators Analysis}

In our previous work, the Aggregator Advisor method ${ }^{12}$ was used to predict putative aggregators and identify known aggregators. The Aggregator Advisor is based on a very simple approach, comprising the calculation of the Tanimoto similarity of the tested compound to a list of $\sim 12600$ know aggregators (using topological fingerprints), and if the similarity is $>0.85$ to at least one of these compounds, and its $\log P$ is $>3$, it is assigned a "possible aggregator" status; in the rest of the cases no prediction is made ("unknown", or "non-aggregator" status). Thus, it is a very conservative approach that is unable to give predictions of compounds lying outside the close structural space of the aggregators list, although it is useful as a fast and easy-to-implement way to identify close analogs to the known aggregators list with high risk of aggregation if lipophilic enough.

On the contrary, the SCAM Detective ${ }^{31}$ is a machine learning-based approach using random forests ${ }^{38}$ that is capable in principle to extrapolate to the whole structural chemical space. The training sets for the SCAM Detective were obtained from pairs of quantitative high-throughput screening (qHTS) campaigns in PubChem ${ }^{39}$ run with the same assay conditions but in the presence and absence of added detergent in the assay buffer. By comparing the dose-response curves in each pair for each compound, in the 
217 presence and in the absence of detergent, it was possible to mark it as putative aggregator or non-aggregator. Models were derived for both AmpC $\beta$-lactamase and the cysteine protease cruzain, which are frequently used counter-assays to discard false positives in assays. ${ }^{40-42}$ The corresponding screens in PubChem were tested at different experimental conditions in terms both of assay buffer and dosing concentrations. This is an interesting feature as by comparing the predictions in both assays an approach to assess the robustness of the prediction is available.

In addition, in the development of the training set, a data-rebalancing approach was applied, so that in both training sets there were equal numbers of aggregators and nonand non-aggregators, and thus have a balanced sensitivity and specificity $(0.72$ and 0.73 , respectively for the AmpC $\beta$-lactamase model, and 0.71 and 0.69 for the cruzain model).

The SCAM Detective also provides a measure of the reliability of its predictions, which is based on the so-called applicability domain $(A D)$ of the model, defined as

$$
D_{\text {cutoff }}=\langle D\rangle+0.5 S
$$

where $\langle D\rangle$ and S are the average and SD of all the Euclidian distances in the descriptor space used between each compound and its nearest neighbors in the training set. New compounds with a minimum distance to the molecules in the training set $D>D_{\text {cutoff }}$ would be outside the AD of the model, meaning that the predictions for it would be in general less reliable. generated for the modeled molecules, aiding to provide an interpretation of the model 
prediction through the groups in the compound most responsible for the aggregating or non-aggregating behavior.

By first applying the Aggregator Advisor method to the updated FooDB, we can identify a total of 92 known aggregators and 37 predicted aggregators. These concentrate mainly in the old FooDB database, since within the new compounds no one was a predicted compound, and only two were known aggregators. These were in the NAG group. By merging known and predicted aggregators, this leads to a $0.56 \%$ and a $0.004 \%$ of aggregator rate for the old and new FooDB molecules, respectively. These small numbers reflect the conservativeness of the Aggregator Advisor and that the novel FooDB molecules display even less overlap with the chemical space of the list of aggregators used by the method compared with the previous FooDB.

These numbers change dramatically upon application of the SCAM Detective. Table 1 displays the total number and percentages of predicted aggregators in both FooDB (and its different subsets) and DrugBank (for comparison purposes) for both the $\beta$-lactamase and cruzain models, as well as their intersection. The actual predictions for all the molecules in FooDB can be obtained in Supporting Information (Table S3). It can be seen that the aggregator rates for the SCAM Detective are much higher. For instance, the $\beta$ lactamase model predicts a $76.7 \%$ of aggregators in FooDB, that rises up to $95.39 \%$ for the AG subset. For the cruzain model, the aggregator rate is $40.82 \%$ for the whole FooDB, and $52.98 \%$ for the AG subset. In both models, the aggregator rates for the new FooDB molecules are significantly higher than for the old ones $(43.17 \%$ and $19.95 \%$, for 
molecules, with long aliphatic chains that makes them prone to aggregation in the AG.

263

264

265

266

267

268

269

270

271

272

273

274

275

277

278

280

281

282

283

As a way of confirmation, Figure 3 displays the predicted fragment contribution maps for FDB00135, a predicted non-aggregator in both models from the old FooDB, and FDB080642, a doubly-predicted aggregator and an AG compound. We can see that while FDB000135 shows green contours indicative of non-aggregation contribution together with some weakly concentrated magenta contours, FDB080642 displays highly concentrated and dark-magenta contours, a signature of high-aggregating contribution, especially in its three polymethylene chains.

On the other hand, the DrugBank display aggregator rates near the old FooDB molecules, slightly lower: $34.44 \%$ and $18.89 \%$ for $\beta$-lactamase and cruzain, respectively.

From Table 1 it can be observed that the $\beta$-lactamase model shows in all the compound sets an increased aggregator rate as compared to the cruzain model. This is in contrast to the original training datasets used, which showed comparable aggregator rates for $\beta$ lactamase and cruzain assays, although obviously the results obtained here depend on the chemical spaces of the molecules that were aggregators in one or the other training sets. In general, there is a highly significant association between the two models, with a $61 \%$ of molecules being aggregators or non-aggregators simultaneously in both models. This rises to $73 \%$ in the case of the DrugBank molecules. By considering the intersection between the models, which would correspond to a more robust (although more conservative) prediction of aggregation, the aggregation rate for the whole FooDB would be $39.25 \%$ (including $15.34 \%, 29.76 \%$ and $52.98 \%$ for the old FooDB, NAG, AG, respectively) vs a $13.32 \%$ for the DrugBank. 
284 If we focus on the reliability of the predictions based on the AD of the different sets, we

285 obtain the results collected in Table 2. Again, the number and percentage of molecules 286 within the AD in one or the other model and the intersection of both, for the different 287 compound sets, are shown. We can see very large percentages of molecules within the 288 applicability domain in the updated FooDB $(81.96 \%$ and $86.57 \%$ for $\beta$-lactamase and 289 cruzain models, respectively), even higher for the new molecules, especially in the case of $A G$, which is very close to $100 \%$ (98.96\% and $99.99 \%$, respectively). For the old FooDB molecules the percentages are lower, $50.93 \%$ and $61.46 \%$; these values are similar to those observed for the DrugBank (52.01\% and 55.93\%).

In this case, the percentage of molecules in the AD is slightly but significantly higher for the cruzain model compared to the $\beta$-lactamase model if we consider the whole set of molecules: $85.64 \%$ vs $81.05 \%$, respectively. The agreement between both models as far as $A D$ is concerned is very high, and $\sim 94 \%$ of the molecules are within or without the $A D$ of both models simultaneously. This is probably due to both models using similar collections of compounds in their training sets, as well as the very high percentages of molecules within the AD in both cases. By intersecting both models, there is just an slight decrease of percentages in all the sets, so that the whole FooDB has a $81.43 \%$ (including 49.51\%, $78.07 \%$, and $98.96 \%$ for old FooDB, NAG, and AG, respectively) vs a $47.58 \%$ for the DrugBank.

We could ask what are the SCAM Detective predictions for the compounds marked as known (92) or predicted (37) aggregators by the Aggregator Advisor. Of the 92 known aggregators, 23 are predicted aggregators by the $\beta$-lactamase model, and 15 by the cruzain model. Of the 37 predicted aggregators, 16 are predicted aggregators by the $\beta$ - 
lactamase model, while 16 are predicted aggregators by the cruzain model. Thus, there seems to be a modest agreement for the prediction of the aggregator class between both SCAM Detective and Aggregator Advisor models, although it must be taken into account that the datasets were derived in different conditions, with different definitions of aggregation (different predicted labels), and with different compound sets. In addition, the numbers used for the comparison are very small, given the tiny number of compounds predicted by the Aggregator Advisor, and we are only considering the aggregators class, not the non-aggregators.

In summary, we have obtained novel predictions for the updated FooDB through the machine learning approach used by the SCAM Detective, which was reported to provide balanced sensitivity/specificity predictions and an increase of accuracy from $46 \%$ to $53 \%^{31}$ compared to other methods (e.g. Aggregator Advisor ${ }^{12}$ and Hit Dexter ${ }^{43}$, see SCAM Detective paper ${ }^{31}$ ). The FooDB show relatively large percentages of predicted aggregated molecules, $76.70 \%$ in $\beta$-lactamase and $40.82 \%$ in cruzain. The old fraction of FooDB displays clearly lower percentages ( $43.17 \%$ and $19.85 \%$, respectively), while the new fraction of molecules shows increased values, especially in the case of AG, which are predicted aggregators in $95.39 \%$ of the cases by the $\beta$-lactamase model and $52.98 \%$ by the cruzain one. For the whole FooDB, a very large proportion of molecules appears to be within the AD of the models, $86.57 \%$ for the cruzain model and $81.96 \%$ for the $\beta$ lactamase. These predictions, provided as Supporting Information (Table S3), are expected to help the community of scientist aiming in understanding the biological mechanisms of action of food compounds to identify aggregators in their assays. 
In our previous work we also analyzed the presence of nuisance substructures in the FooDB. In this section we repeat that analysis for the updated FooDB. Three filter sets were used, namely PAINS, Glaxo, and LINT. The first one of these was derived by Baell and Holloway ${ }^{22}$ after analysis of a series of high-throughput screens run with the AlphaScreen technology, and comprise a total of 481 filters, grouped in three families with decreasing statistical support: family A (16 filters), corresponding to the filters with comprising 409 filters with the lowest statistical support. The PAINS filters were derived using a relatively "clean" screening collection developed after an in-silico effort to preclude the presence of inappropriately reactive functional groups, like epoxides, aziridines, alkyl halides, labile esters, etc. ${ }^{22}$ Thus, in order to be able in our analysis for the detection of these substructures, we also included two additional more basic filter sets: Glaxo, corresponding to 55 filters derived in GlaxoWellcome, ${ }^{19}$ and LINT, of 57 filters and generated in Pfizer. ${ }^{20}$

Figure 4 displays the 18 PAINS filters matched by at least one compound in FooDB, color coded by filter family (green for family A, blue for family B, and red for family C). We can see the same set of filters and almost the same distribution as the one observed previously, ${ }^{29}$ dominated by "catechol_A(92)", followed by "quinone_A(370)", 348 "imine_one_A(321)" and "azo_A(324)". No molecules in the updated FooDB match any more of the 18 matching filters in the old FooDB. The reason is that none of the AG 350 molecules match any of these filters, while just a few set of 74 NAG match a reduced set 351 of three filters observed before:29 "catechol_A(92)", "imine_one_A(321)", and 352 "quinone_A(370)". Because of this, the percentage of molecules filtered by PAINS filters 
353 is reduced from $6.80 \%$ down to $2.23 \%$. If we focus on the most reliable family $A$, the percentage of filtered molecules is just a $0.37 \%$ (before it was $1.11 \%$ ), corresponding to $\sim 17 \%$ of the total of matches by the PAINS set, while there are 6 filters in this family that match at least one molecule.

Figure 5 shows the matches distribution for the Glaxo filter set. 35 filters match at least one molecule, and now the distribution is overwhelmingly dominated by the first filter, namely "I1 Aliphatic methylene chains 7 or more long", with a total of 51597 matches. This is because of the new AG molecules, almost all of them matching this filter, as expected due to the frequent presence in their structure of long polymethylenic chains.

Other than that, the AG do not match any other Glaxo filter. In the case of the NAG molecules, again the most frequent filter is "I1 Aliphatic methylene chains 7 or more long", but in this case the second one is no longer "N3 Saponin derivatives", which is very unusual in these molecules, but instead "I15 Di and Triphosphates", followed by "I5 Thiols" and "N2 Polyenes". All in all, no additional Glaxo filter absent in the previous study $^{29}$ matches any of the new FooDB molecules.

As regarding the LINT filters (Figure 6), a large increase of matched molecules is observed for the first two filters, "long aliphatic chain, 6+" and "aliphatic ester, not lactones", as compared to the matches in the previous FooDB ${ }^{29}$ due again to the large number of new AG molecules that match these substructures. No additional filter is matched by the AG compounds, while the NAG ones has as most frequently matched filter "S/PO3 groups", followed by the previous "Iong aliphatic chain, 6+", "alkyl esters of S or P" and "aliphatic ester, not lactones". As was observed with PAINS and Glaxo, no new filter appears here that were not present in the previous study. 
376 Table 3 collects the filter match statistics for all these filter sets. We can see that, as said

377 before, the new compounds decrease the stringency of both the PAINS and PAINS-A

378 sets, due to the little number of matching molecules in the new FooDB molecules for

379 these sets (74 in total, all of them in the NAG group). The effect of this is to reduce the

380 percentage of matched molecules from $6.80 \%$ to $2.23 \%$ (PAINS), and from $1.11 \%$ to

$3810.37 \%$ (PAINS-A). The contrary is observed for Glaxo and LINT, where the percentage of

382 filtered molecules raises up to $78.45 \%$ and $85.11 \%$, respectively, while in the previous

383 work it was $36.18 \%$ and $55.43 \%$. Similar dual effect is observed if we focus on the

384 normalized percentage of matched molecules: now it is $0.0046 \%, 0.023 \%, 1.44 \%$, and

1.49\% for PAINS, PAINS-A, Glaxo, and LINT, while before it was $0.014 \%, 0.069 \%, 0.658 \%$, and $0.973 \%$. As in our previous work, the stringency order considering the percentage of filtered molecules is as follows: PAINS-A $<$ PAINS $<$ Glaxo $<$ LINT. If instead we consider this percentage but normalized by the number of filters in the set, PAINS and PAINS-A switch order, but the rest remains the same: PAINS < PAINS-A < Glaxo < LINT. The same order is observed by the fraction of filters with at least one matching molecule in each set, that goes from 18 out of 481 in PAINS, to 49 out of 57 in the case of LINT, with 35 out of 55 in the case of Glaxo in between.

All these filter matches are collected in Supporting Information (Table S4). In the same way, it is expected that this will be useful to identify interferences in biochemical and biological assays of food compounds. 
A large research effort is being devoted to the determination of the biological mechanism of action of food compounds, in order to understand the beneficial or harmful effect of foods in human health. ${ }^{1-9,44}$ These studies are conducted by performing biochemical or biological (cellular) assays aiming to see if the food molecule interacts with some biological target, typically a protein. It is well known from the field of drug discovery that in some cases an assay can result in a false positive or misleading outcome due to some property of the tested molecule: ${ }^{10,24,26-28,41}$ the molecule forms colloidal aggregates that denature the target protein or has some substructure that make it prone to membrane disruption, (photo)reactivity, redox cycling, etc., or rather to generate interferences with the assay signal. ${ }^{18-23,26}$

In a previous work, ${ }^{29}$ we applied cheminformatic techniques from the drug discovery field to identify molecules prone to such false-positive behavior in a database of food compounds, the FooDB, ${ }^{30}$ to find food compounds with these putative issues. The FooDB is (quoting from its web site) "the world's largest and most comprehensive resource on food constituents, chemistry and biology. It provides information on both macronutrients and micronutrients, including many of the constituents that give foods their flavor, color, taste, texture and aroma". Here we provide an update of that analysis after the FooDB more than duplicated its size ( $26 \mathrm{~K}$ compounds to $\sim 71 \mathrm{~K}$ compounds), that includes also the use of novel machine learning models to predict aggregation, as

417 the method used before (Aggregator Advisor ${ }^{12}$ ) was not able to give predictions for the majority of food compounds. We opted to use the so-called SCAM Detective, ${ }^{31}$ as it has been observed to yield accuracies $\sim 50 \%$ above previous methods, including the 
420 Aggregator Advisor, and as other methods like the Hit Dexter ${ }^{43}$ show lower accuracy ${ }^{31}$

421 and also predict a slightly different endpoint, namely hit promiscuity, which is a related

422 but different label (aggregators are all promiscuous, but not all promiscuous compounds

423 are such because of aggregating behavior).

424 From a practical point of view, the files provided here as Supplementary Information

425 should be used by the experimenter to find if the tested compound appears there with one or more annotations for aggregation and/or interference. If the annotation is for

427 aggregation, its presence can be experimentally checked by different approaches: decrease of activity after addition of small quantities of non-ionic detergents, counterassay in aggregation-sensitive assays (e.g. $\beta$-lactamase), or detection of colloidal aggregates through dynamic light scattering. On the other hand, if the annotation is for a substructure that generates assay signal interferences (e.g. absorption, fluorescence, etc.), a possible solution is the test in an orthogonal assay using an alternative technology and signal. The third option are those interfering substructures that provide nonspecific activity (promiscuity) through variable mechanisms (e.g. membrane disruption); in that case it could be possible to run a counter-assay with a different and unrelated target and the same technology. In general, it is always advisable to check in public databases like ChEMBL ${ }^{45}$ or PubChem ${ }^{39}$ about previously reported activities of the compound, which would be informative about possible promiscuity issues if the molecule has shown activity against a wide set of unrelated targets. In addition, if 440 chemical modifications are performed on the compound, finding a lack of a defined 441 structure-activity relationship would be a signature of artifactual activity. More 
442 thorough approaches to these issues have been described elsewhere; ${ }^{46-48}$ and for more 443 specifically referring to publication in ACS journals see reference (28).

444 The updated prediction performed in this work has increased considerably the number of putative aggregators in FooDB: $77 \%$ for the $\beta$-lactamase model and $41 \%$ for the cruzain model. One reason is the AG component of the new FooDB molecules, for which the $\beta$-lactamase model predicts a $95 \%$ of aggregators, and the cruzain a $53 \%$ (Table 2 ). Given the very large hydrophobicity of these molecules (median logP of 17.9) together with the extreme flexibility of their aliphatic structure, these predictions seem quite reasonable. The higher hydrophobicity, could also contribute to the increase in aggregator rates in NAG molecules over the old FooDB molecules, together with the significantly larger surface areas of the former group as compared to the latter and the higher number of rotatable bonds. In addition, another reason for getting many more aggregators is that the Aggregator Advisor had not been able to give predictions for the vast majority of the FooDB molecules as just a few of them were similar to one or more in the list of known aggregators: using a Tanimoto radius of 0.85 , only 437 in the old FooDB, 20 for NAG, and none for the AG. This shows the advantages of the SCAM Detective, that is trained with a very large and diverse dataset of both aggregators and non-aggregators and gives predictions for the whole chemical space. Also, it is worth mentioning that a very large fraction of the FooDB molecules are within the AD of the SCAM Detective models, for which the predictions are expected to be more reliable, 462 ranging from $51 \%$ to $100 \%$ depending on the subset and model. Thus, we expect this effort to give a much more reliable and comprehensive identification of putative aggregators in the food molecules of FooDB. 
465 As regarding the interference filter analysis, in the previous work we applied as

466 cheminformatic tool three well-known substructure filter sets derived from the high-

467 throughput screening and medicinal chemistry fields: PAINS, ${ }^{22}$ Glaxo ${ }^{19}$ and LINT. ${ }^{20}$ Here

468 we have applied these filters to the updated FooDB too. As a result, for the PAINS we

469 have observed a very small number of novel FooDB molecules matching them, so that

470 the observed current distribution is very similar to the previous one; also the set of

471 matched filters remains equal. The same set of matched filters are also observed again

472 with the Glaxo and LINT filters, but in this case the distributions change significantly as

473 the filters representing long aliphatic chains or aliphatic esters ("I1 Aliphatic methylene

474 chains 7 or more long" in Glaxo and "long aliphatic chain, $6+$ " and "aliphatic ester, not

475 lactones" in LINT) have an enormous increase of hits, corresponding mostly to the new

476 AG compounds. As a result, the interference rates for these filter sets increase

477 considerably from the previous analysis, resulting in a total of $78 \%$ and $85 \%$ for Glaxo

478 and LINT, respectively (before they were $36 \%$ and $55 \%$ ).

479 In general, we observe a decrease of drug-like properties in the new FooDB molecules,

480 with a significant decrease of its drug-like "chemical beauty" (as measured by the

481 Estimation of Drug-likeness (QED) descriptor ${ }^{35}, p$-value $<0.001$ in post-hoc test, see

482 Supplementary Material, Table S2) and an increase of aggregator and interference rates

483 (for the Glaxo and LINT filters). The new FooDB molecules (especially the AG component)

484 tend to have more hydrophobicity, flexibility, and molecular weight; these factors make

485 them more prone to aggregation on one hand (also the higher TPSA in the NAG

486 molecules), and to display the long polymethylene-type of interfering substructure

487 appearing in the Glaxo and LINT filter sets. The near complete absence of interferences 
of the PAINS filters can on the other hand be explained by considering that the later were derived from a collection of relatively "clean" compounds previously filtered from more basic problematic substructures, to make them more amenable as starting points for drug development. The PAINS filters derived from that collection would be more 492 specific to drug- or lead-like molecules, probably of a more synthetic origin, and corresponding to substructures scarcely present in the FooDB.

To conclude, we can say that the putative aggregators and interference matches for the new FooDB found in this work and available as Supporting Information would help to decrease the false positives in assays performed with food compounds, by applying, when present, the approaches discussed above, and therefore to gain a better and more robust understanding of their biological mechanisms of action, thus reducing the rates of false positive results in public databases like ChEMBL ${ }^{45}$ or PubChem ${ }^{39}$. Other uses for these predictions would be the prioritization of compounds for testing, applications in large-scale data mining efforts for understanding structure-activity relationships, design of reliable nutraceutics, and selection of novel scaffolds for development of new drugs. 
505 American Chemical Society (ACS), Small, Colloidal, Interfering Molecules (SCAM) 506 Detective, Pan-Assay INterference compoundS (PAINS), Invalid Metabolic PanaceaS 507 (IMPs)

\section{AUTHOR INFORMATION}

509 Corresponding Author:

510 Gonzalo Colmenarejo - Biostatistics and Bioinformatics Unit. IMDEA Food, CEI

511 UAM+CSIC, E28049 Madrid, Spain. orcid.org/0000-0002-8249-4547.

512 gonzalo.colmenarejo@imdea.org

513 Authors:

514 Andrés Sánchez-Vicente - Biostatistics and Bioinformatics Unit. IMDEA Food, CEI

515 UAM+CSIC, E28049 Madrid, Spain

$516 \quad$ Notes

517 The authors declare no competing financial interest.

518

519 ACKNOWLEDGEMENTS

520 The Dr Wishart Research Group is thanked for providing the updated version of FooDB

521 in SDF format. Dr Patrick Walters is acknowledged for providing the Glaxo, LINT and 522 PAINS nuisance filters in SMARTS format adapted for RDKIT. 
525 Statistical Analysis of PhysChem Distributions.xlsx. Both p.values for tests for

526 comparison of physicochemical distributions between groups of compounds (omnibus

527 and pairwise post-hoc), and descriptive statistics (median + interquartile range) for

528 these distributions are collected here.

529 SCAM Detective Predictions for FooDB.xIsx Aggregator predictions for the FooDB using

530 the SCAM Detective (both $\beta$-lactamase and cruzain models). The prediction result (0

531 non-aggregator, 1 aggregator) plus the AD result (Inside AD vs Outside AD) is shown for

532 each FooDB compound.

533 Filter Matches for FooDB.xIsx. Filter matches for PAINS, Glaxo and LINT filter sets for 534 the updated FooDB

535

536 
538 (1) Polya, G. M. Biochemical Targets of Plant Bioactive Compounds: A Pharmacological Reference Guide to Sites of Action and Biological Effects; Taylor \& Francis: London; New York, 2003.

(2) Hu, J.; Wang, J.; Gan, Q.; Ran, Q.; Lou, G.; Xiong, H.; Peng, C.; Sun, J.; Yao, R.; Huang, Q. Impact of Red Yeast Rice on Metabolic Diseases: A Review of Possible Mechanisms of Action. J. Agric. Food Chem. 2020, 68 (39), 10441-10455. https://doi.org/10.1021/acs.jafc.0c01893.

(3) Teodoro, A. J. Bioactive Compounds of Food: Their Role in the Prevention and Treatment of Diseases. Oxidative Medicine and Cellular Longevity 2019, 2019, 14. https://doi.org/10.1155/2019/3765986.

(4) Mbachu, O. C.; Howell, C.; Simmler, C.; Malca Garcia, G. R.; Skowron, K. J.; Dong, H.; Ellis, S. G.; Hitzman, R. T.; Hajirahimkhan, A.; Chen, S.-N.; Nikolic, D.; Moore, T. W.; Vollmer, G.; Pauli, G. F.; Bolton, J. L.; Dietz, B. M. SAR Study on Estrogen Receptor $\alpha / \beta$ Activity of (Iso)Flavonoids: Importance of Prenylation, C-Ring (Un)Saturation, and Hydroxyl Substituents. J. Agric. Food Chem. 2020, 68 (39), 10651-10663. https://doi.org/10.1021/acs.jafc.0c03526.

(5) Perez-Gregorio, R.; Simal-Gandara, J. A Critical Review of Bioactive Food Components, and of Their Functional Mechanisms, Biological Effects and Health Outcomes. Current Pharmaceutical Design 2017, 23 (19), 2731-2741.

(6) Sharifi-Rad, J.; Quispe, C.; Zam, W.; Kumar, M.; Cardoso, S. M.; Pereira, O. R.; Ademiluyi, A. O.; Adeleke, O.; Moreira, A. C.; Živković, J.; Noriega, F.; Ayatollahi, S. A.; Kobarfard, F.; Faizi, M.; Martorell, M.; Cruz-Martins, N.; Butnariu, M.; Bagiu, I. C.; Bagiu, R. V.; Alshehri, M. M.; Cho, W. C. Phenolic Bioactives as Antiplatelet Aggregation Factors: The Pivotal Ingredients in Maintaining Cardiovascular Health. Oxidative Medicine and Cellular Longevity 2021, 2021, e2195902. https://doi.org/10.1155/2021/2195902.

(7) Sharifi-Rad, J.; Cruz-Martins, N.; López-Jornet, P.; Lopez, E. P.-F.; Harun, N.; Yeskaliyeva, B.; Beyatli, A.; Sytar, O.; Shaheen, S.; Sharopov, F.; Taheri, Y.; Docea, A. O.; Calina, D.; Cho, W. C. Natural Coumarins: Exploring the Pharmacological Complexity and Underlying Molecular Mechanisms. Oxidative Medicine and Cellular Longevity 2021, 2021, e6492346. https://doi.org/10.1155/2021/6492346.

(8) Gry, J.; Black, L.; Eriksen, F. D.; Pilegaard, K.; Plumb, J.; Rhodes, M.; Sheehan, D.; Kiely, M.; Kroon, P. A. EuroFIR-BASIS - a Combined Composition and Biological Activity Database for Bioactive Compounds in Plant-Based Foods. Trends in Food Science \& Technology 2007, 18 (8), 434-444. https://doi.org/10.1016/j.tifs.2007.05.008.

(9) Faudone, G.; Arifi, S.; Merk, D. The Medicinal Chemistry of Caffeine. J. Med. Chem. 2021. https://doi.org/10.1021/acs.jmedchem.1c00261.

(10) McGovern, S. L.; Caselli, E.; Grigorieff, N.; Shoichet, B. K. A Common Mechanism Underlying Promiscuous Inhibitors from Virtual and High-Throughput Screening. J. Med. Chem. 2002, 45 (8), 1712-1722. https://doi.org/10.1021/jm010533y.

(11) Reker, D.; Bernardes, G. J. L.; Rodrigues, T. Computational Advances in Combating Colloidal Aggregation in Drug Discovery. Nat. Chem. 2019, 11 (5), 402-418. https://doi.org/10.1038/s41557-019-0234-9. 
(12) Irwin, J. J.; Duan, D.; Torosyan, H.; Doak, A. K.; Ziebart, K. T.; Sterling, T.; Tumanian, G.; Shoichet, B. K. An Aggregation Advisor for Ligand Discovery. J. Med. Chem. 2015, 58 (17), 7076-7087. https://doi.org/10.1021/acs.jmedchem.5b01105.

(13) Feng, B. Y.; Simeonov, A.; Jadhav, A.; Babaoglu, K.; Inglese, J.; Shoichet, B. K.; Austin, C. P. A High-Throughput Screen for Aggregation-Based Inhibition in a Large Compound Library. J. Med. Chem. 2007, 50 (10), 2385-2390. https://doi.org/10.1021/jm061317y.

(14) Owen, S. C.; Doak, A. K.; Ganesh, A. N.; Nedyalkova, L.; McLaughlin, C. K.; Shoichet, B. K.; Shoichet, M. S. Colloidal Drug Formulations Can Explain "BellShaped" Concentration-Response Curves. ACS Chem. Biol. 2014, 9 (3), 777-784. https://doi.org/10.1021/cb4007584.

(15) Coan, K. E. D.; Shoichet, B. K. Stoichiometry and Physical Chemistry of Promiscuous Aggregate-Based Inhibitors. J. Am. Chem. Soc. 2008, 130 (29), 9606-9612. https://doi.org/10.1021/ja802977h.

(16) Coan, K. E. D.; Maltby, D. A.; Burlingame, A. L.; Shoichet, B. K. Promiscuous Aggregate-Based Inhibitors Promote Enzyme Unfolding. J. Med. Chem. 2009, 52 (7), 2067-2075. https://doi.org/10.1021/jm801605r.

(17) Zorn, J. A.; Wille, H.; Wolan, D. W.; Wells, J. A. Self-Assembling Small Molecules Form Nanofibrils That Bind Procaspase-3 to Promote Activation. J Am Chem Soc 2011, 133 (49), 19630-19633. https://doi.org/10.1021/ja208350u.

(18) Rishton, G. M. Reactive Compounds and in Vitro False Positives in HTS. Drug Discovery Today 1997, 2 (9), 382-384. https://doi.org/10.1016/S13596446(97)01083-0.

(19) Hann, M.; Hudson, B.; Lewell, X.; Lifely, R.; Miller, L.; Ramsden, N. Strategic Pooling of Compounds for High-Throughput Screening. J. Chem. Inf. Comput. Sci. 1999, 39 (5), 897-902. https://doi.org/10.1021/ci990423o.

(20) Blake, J. F. Identification and Evaluation of Molecular Properties Related to Preclinical Optimization and Clinical Fate. Med Chem 2005, 1 (6), 649-655. https://doi.org/10.2174/157340605774598081.

(21) Brenk, R.; Schipani, A.; James, D.; Krasowski, A.; Gilbert, I. H.; Frearson, J.; Wyatt, P. G. Lessons Learnt from Assembling Screening Libraries for Drug Discovery for Neglected Diseases. ChemMedChem 2008, 3 (3), 435-444. https://doi.org/10.1002/cmdc.200700139.

(22) Baell, J. B.; Holloway, G. A. New Substructure Filters for Removal of Pan Assay Interference Compounds (PAINS) from Screening Libraries and for Their Exclusion in Bioassays. J. Med. Chem. 2010, 53 (7), 2719-2740. https://doi.org/10.1021/jm901137j.

(23) Baell, J. B. Feeling Nature's PAINS: Natural Products, Natural Product Drugs, and Pan Assay Interference Compounds (PAINS). J. Nat. Prod. 2016, 79 (3), 616-628. https://doi.org/10.1021/acs.jnatprod.5b00947.

(24) Baell, J. B.; Nissink, J. W. M. Seven Year Itch: Pan-Assay Interference Compounds (PAINS) in 2017-Utility and Limitations. ACS Chem. Biol. 2018, 13 (1), 36-44. https://doi.org/10.1021/acschembio.7b00903.

(25) Chakravorty, S. J.; Chan, J.; Greenwood, M. N.; Popa-Burke, I.; Remlinger, K. S.; Pickett, S. D.; Green, D. V. S.; Fillmore, M. C.; Dean, T. W.; Luengo, J. I.; Macarrón, R. Nuisance Compounds, PAINS Filters, and Dark Chemical Matter in 
the GSK HTS Collection. SLAS Discov 2018, 23 (6), 532-545. https://doi.org/10.1177/2472555218768497.

(26) Dahlin, J. L.; Auld, D. S.; Rothenaigner, I.; Haney, S.; Sexton, J. Z.; Nissink, J. W. M.; Walsh, J.; Lee, J. A.; Strelow, J. M.; Willard, F. S.; Ferrins, L.; Baell, J. B.; Walters, M. A.; Hua, B. K.; Hadian, K.; Wagner, B. K. Nuisance Compounds in Cellular Assays. Cell Chemical Biology 2021, 28 (3), 356-370. https://doi.org/10.1016/j.chembiol.2021.01.021.

(27) Baell, J.; Walters, M. A. Chemistry: Chemical Con Artists Foil Drug Discovery. Nature News 2014, 513 (7519), 481. https://doi.org/10.1038/513481a.

(28) Aldrich, C.; Bertozzi, C.; Georg, G. I.; Kiessling, L.; Lindsley, C.; Liotta, D.; Merz, K. M.; Schepartz, A.; Wang, S. The Ecstasy and Agony of Assay Interference Compounds. ACS Cent. Sci. 2017, 3 (3), 143-147. https://doi.org/10.1021/acscentsci.7b00069.

(29) Kaya, I.; Colmenarejo, G. Analysis of Nuisance Substructures and Aggregators in a Comprehensive Database of Food Chemical Compounds. J. Agric. Food Chem. 2020, 68 (33), 8812-8824. https://doi.org/10.1021/acs.jafc.0c02521.

(30) FooDB https://foodb.ca/ (accessed 2021 -09-13).

(31) Alves, V. M.; Capuzzi, S. J.; Braga, R. C.; Korn, D.; Hochuli, J. E.; Bowler, K. H.; Yasgar, A.; Rai, G.; Simeonov, A.; Muratov, E. N.; Zakharov, A. V.; Tropsha, A. SCAM Detective: Accurate Predictor of Small, Colloidally Aggregating Molecules. J. Chem. Inf. Model. 2020, 60 (8), 4056-4063. https://doi.org/10.1021/acs.jcim.0c00415.

(32) RDKit: Open-source cheminformatics https://www.rdkit.org/ (accessed 2021 -09 $-03)$.

(33) Bento, A. P.; Hersey, A.; Félix, E.; Landrum, G.; Gaulton, A.; Atkinson, F.; Bellis, L. J.; De Veij, M.; Leach, A. R. An Open Source Chemical Structure Curation Pipeline Using RDKit. Journal of Cheminformatics 2020, 12 (1), 51. https://doi.org/10.1186/s13321-020-00456-1.

(34) Wishart, D. S.; Knox, C.; Guo, A. C.; Shrivastava, S.; Hassanali, M.; Stothard, P.; Chang, Z.; Woolsey, J. DrugBank: A Comprehensive Resource for in Silico Drug Discovery and Exploration. Nucleic Acids Research 2006, 34 (suppl_1), D668D672. https://doi.org/10.1093/nar/gkj067.

(35) Bickerton, G. R.; Paolini, G. V.; Besnard, J.; Muresan, S.; Hopkins, A. L. Quantifying the Chemical Beauty of Drugs. Nature Chem 2012, 4 (2), 90-98. https://doi.org/10.1038/nchem.1243.

(36) Bemis, G. W.; Murcko, M. A. The Properties of Known Drugs. 1. Molecular Frameworks. J. Med. Chem. 1996, 39 (15), 2887-2893. https://doi.org/10.1021/jm9602928.

(37) Bemis, G. W.; Murcko, M. A. Properties of Known Drugs. 2. Side Chains. J. Med. Chem. 1999, 42 (25), 5095-5099. https://doi.org/10.1021/jm9903996.

(38) Breiman, L. Random Forest. Machine Learning 2001, 45, 5-32.

(39) Kim, S.; Chen, J.; Cheng, T.; Gindulyte, A.; He, J.; He, S.; Li, Q.; Shoemaker, B. A.; Thiessen, P. A.; Yu, B.; Zaslavsky, L.; Zhang, J.; Bolton, E. E. PubChem in 2021: New Data Content and Improved Web Interfaces. Nucleic Acids Research 2021, 49 (D1), D1388-D1395. https://doi.org/10.1093/nar/gkaa971. 
(40) Feng, B. Y.; Shelat, A.; Doman, T. N.; Guy, R. K.; Shoichet, B. K. High-Throughput Assays for Promiscuous Inhibitors. Nat Chem Biol 2005, 1 (3), 146-148. https://doi.org/10.1038/nchembio718.

(41) Jadhav, A.; Ferreira, R. S.; Klumpp, C.; Mott, B. T.; Austin, C. P.; Inglese, J.; Thomas, C. J.; Maloney, D. J.; Shoichet, B. K.; Simeonov, A. Quantitative Analyses of Aggregation, Autofluorescence, and Reactivity Artifacts in a Screen for Inhibitors of a Thiol Protease. J. Med. Chem. 2010, 53 (1), 37-51. https://doi.org/10.1021/jm901070c.

(42) Mott, B. T.; Ferreira, R. S.; Simeonov, A.; Jadhav, A.; Ang, K. K.-H.; Leister, W.; Shen, M.; Silveira, J. T.; Doyle, P. S.; Arkin, M. R.; McKerrow, J. H.; Inglese, J.; Austin, C. P.; Thomas, C. J.; Shoichet, B. K.; Maloney, D. J. Identification and Optimization of Inhibitors of Trypanosomal Cysteine Proteases: Cruzain, Rhodesain, and TbCatB. J. Med. Chem. 2010, 53 (1), 52-60. https://doi.org/10.1021/jm901069a.

(43) Stork, C.; Chen, Y.; Šícho, M.; Kirchmair, J. Hit Dexter 2.0: Machine-Learning Models for the Prediction of Frequent Hitters. J. Chem. Inf. Model. 2019, 59 (3), 1030-1043. https://doi.org/10.1021/acs.jcim.8b00677.

(44) Chen, M.; Pan, D.; Zhou, T.; Gao, X.; Dang, Y. Novel Umami Peptide IPIPATKT with Dual Dipeptidyl Peptidase-IV and Angiotensin I-Converting Enzyme Inhibitory Activities. J. Agric. Food Chem. 2021, 69 (19), 5463-5470. https://doi.org/10.1021/acs.jafc.0c07138.

(45) Gaulton, A.; Hersey, A.; Nowotka, M.; Bento, A. P.; Chambers, J.; Mendez, D.; Mutowo, P.; Atkinson, F.; Bellis, L. J.; Cibrián-Uhalte, E.; Davies, M.; Dedman, N.; Karlsson, A.; Magariños, M. P.; Overington, J. P.; Papadatos, G.; Smit, I.; Leach, A. R. The ChEMBL Database in 2017. Nucleic Acids Res 2017, 45 (Database issue), D945-D954. https://doi.org/10.1093/nar/gkw1074.

(46) Seidler, J.; McGovern, S. L.; Doman, T. N.; Shoichet, B. K. Identification and Prediction of Promiscuous Aggregating Inhibitors among Known Drugs. J. Med. Chem. 2003, 46 (21), 4477-4486. https://doi.org/10.1021/jm030191r.

(47) Auld, D. S.; Inglese, J.; Dahlin, J. L. Assay Interference by Aggregation. In Assay Guidance Manual; Markossian, S., Grossman, A., Brimacombe, K., Arkin, M., Auld, D., Austin, C. P., Baell, J., Chung, T. D. Y., Coussens, N. P., Dahlin, J. L., Devanarayan, V., Foley, T. L., Glicksman, M., Hall, M. D., Haas, J. V., Hoare, S. R. J., Inglese, J., Iversen, P. W., Kales, S. C., Lal-Nag, M., Li, Z., McGee, J., McManus, O., Riss, T., Saradjian, P., Sittampalam, G. S., Tarselli, M., Trask, O. J., Wang, Y., Weidner, J. R., Wildey, M. J., Wilson, K., Xia, M., Xu, X., Eds.; Eli Lilly \& Company and the National Center for Advancing Translational Sciences: Bethesda (MD), 2004.

(48) Coussens, N. P.; Auld, D. S.; Thielman, J. R.; Wagner, B. K.; Dahlin, J. L. Addressing Compound Reactivity and Aggregation Assay Interferences: Case Studies of Biochemical High-Throughput Screening Campaigns Benefiting from the National Institutes of Health Assay Guidance Manual Guidelines. SLAS Discov 2021, 24725552211026240 . https://doi.org/10.1177/24725552211026239. 
721 AS-R acknowledges the Consejería de Ciencia, Universidades e Innovación de la

722 Comunidad de Madrid, Spain (Ref. PEJ-2020-Al/BMD-19384), for a research assistant 723 contract.

724 


\section{FIGURE CAPTIONS}

1. Figure 1. Boxplots for distributions of TPSA (topological polar surface area), LOGP (logarithm of the octanol/water partition coefficient), RB (number of rotatable bonds), HBD (number of hydrogen bond donors), HBA (number of hydrogen bond acceptors), MW (molecular weight), QED (quantitative estimation of druglikeness), NRING (number of rings), and FSP3 (fraction of sp3-hybridized carbons), for the previous release of FooDB analyzed before ${ }^{29}$ (FDB(OLD)), new non-acylglycerol FooDB molecules in the new release (FDB(NEW/NAG)), new FooDB acylglycerol molecules (FDB(NEW/AG)), and DrugBank molecules (DB). For clarity purposes, outliers have been removed from the plots.

2. Figure 2. Bemis-Murcko ${ }^{36,37}$ scaffold distributions ( 10 top scaffolds only shown in decreasing frequency) for old FooDB, new FooDB (NAG), and DrugBank molecules.

3. Figure 3. Fragment contribution maps for SCAM Detective predictions for FDB00135 (from the former FooDB) and FDB080642 (a novel AG) in the $\beta$ lactamase and cruzain models. The former compound is a predicted nonaggregator in both models, while the later is a predicted aggregator in both models. The color and concentration of contours indicate the direction and strength of the contribution: magenta for aggregation, and green for nonaggregation. Strong contributions result in concentrated contours, weak in separated ones.

4. Figure 4. PAINS filter set distribution across FooDB matching molecules. Only the 18 filters with at least one match are displayed. Bars are color coded by filter family, where family $A$ is green, family $B$ is blue, and family $C$ is red. 
5. Figure 5. Glaxo filter set distribution across the FooDB matching molecules. Only the 35 filters with at least one match are displayed.

752

6. Figure 6. LINT filter set distribution across the FooDB matching molecules. Only the 49 filters with at least one match are displayed.

754 
Table 1. Statistics of Prediction of Aggregators by the SCAM Detective ${ }^{a}$

\begin{tabular}{|c|c|c|c|}
\hline Compound Set & $\beta$-lactamase & cruzain & Both \\
\hline FooDB & 54147 (76.70) & $28815(40.82)$ & 27707 (39.25) \\
\hline FooDB(OLD) & $10124(43.17)$ & 4656 (19.85) & $3598(15.34)$ \\
\hline FooDB(NEW/NAG) & 2608 (69.94) & $1160(31.11)$ & $1110(29.76)$ \\
\hline FooDB(NEW/AG) & 41415 (95.39) & 22999 (52.98) & 22999 (52.98) \\
\hline DrugBank & $755(34.44)$ & $414(18.89)$ & $292(13.32)$ \\
\hline
\end{tabular}


Table 2. Molecules Within Applicability Domain of SCAM Detective Models ${ }^{\mathrm{a}}$

\begin{tabular}{cccc}
\hline Compound Set & $\boldsymbol{\beta}$-lactamase & cruzain & Both \\
\hline FooDB & $57859(81.96)$ & $61113(86.57)$ & $57487(81.43)$ \\
FooDB(OLD) & $11945(50.93)$ & $14415(61.46)$ & $11611(49.51)$ \\
FooDB(NEW/NAG) & $2950(79.11)$ & $3288(88.17)$ & $2912(78.07)$ \\
FooDB(NEW/AG) & $42964(98.96)$ & $43410(99.99)$ & $42964(98.96)$ \\
DrugBank & $1140(52.01)$ & $1226(55.93)$ & $1043(47.58)$ \\
\hline
\end{tabular}

${ }^{a}$ For the different compound sets, the number (percentage) of compounds within the applicability domain in the $\beta$-lactamase and cruzain models, and the intersection of both models, are shown. 
Table 3. Statistics of Matches of Filter Sets for FooDB ${ }^{a}$

\begin{tabular}{|c|c|c|c|c|c|}
\hline Filter Set & \# filters & $\begin{array}{l}\text { \# matching } \\
\text { filters }\end{array}$ & $\begin{array}{l}\text { \# matching } \\
\text { molecules }\end{array}$ & $\begin{array}{l}\text { Filtered } \\
\text { molecules } \\
(\%)\end{array}$ & $\begin{array}{l}\text { Filtered } \\
\text { molecules } \\
\text { / filter (\%) }\end{array}$ \\
\hline PAINS & 481 & 18 & 1554 & 2.23 & 0.0046 \\
\hline PAINS-A & 16 & 6 & 260 & 0.37 & 0.023 \\
\hline Glaxo & 55 & 35 & 54693 & 78.45 & 1.44 \\
\hline LINT & 57 & 49 & 59337 & 85.11 & 1.49 \\
\hline
\end{tabular}



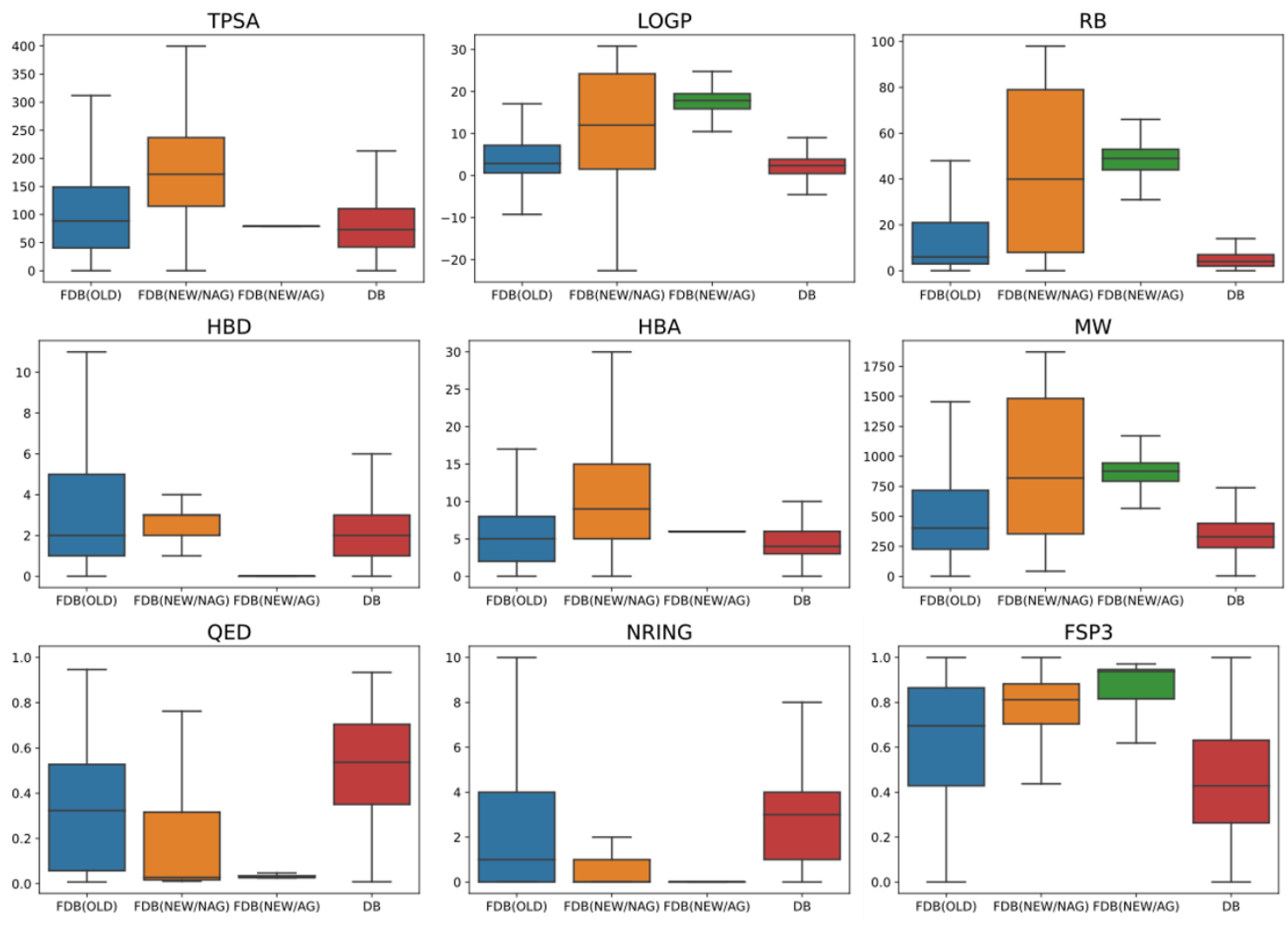

Figure 1. 


\section{FDB (OLD)}

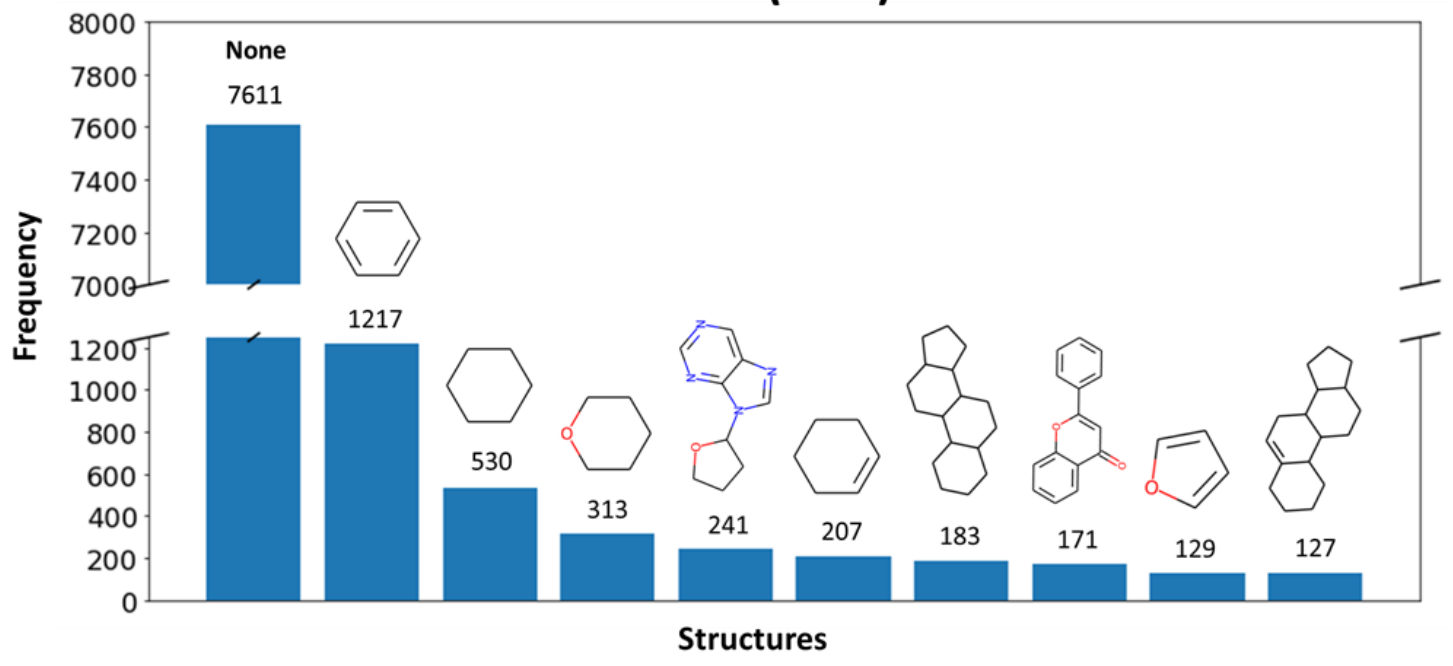

FDB (NEW/NAG)

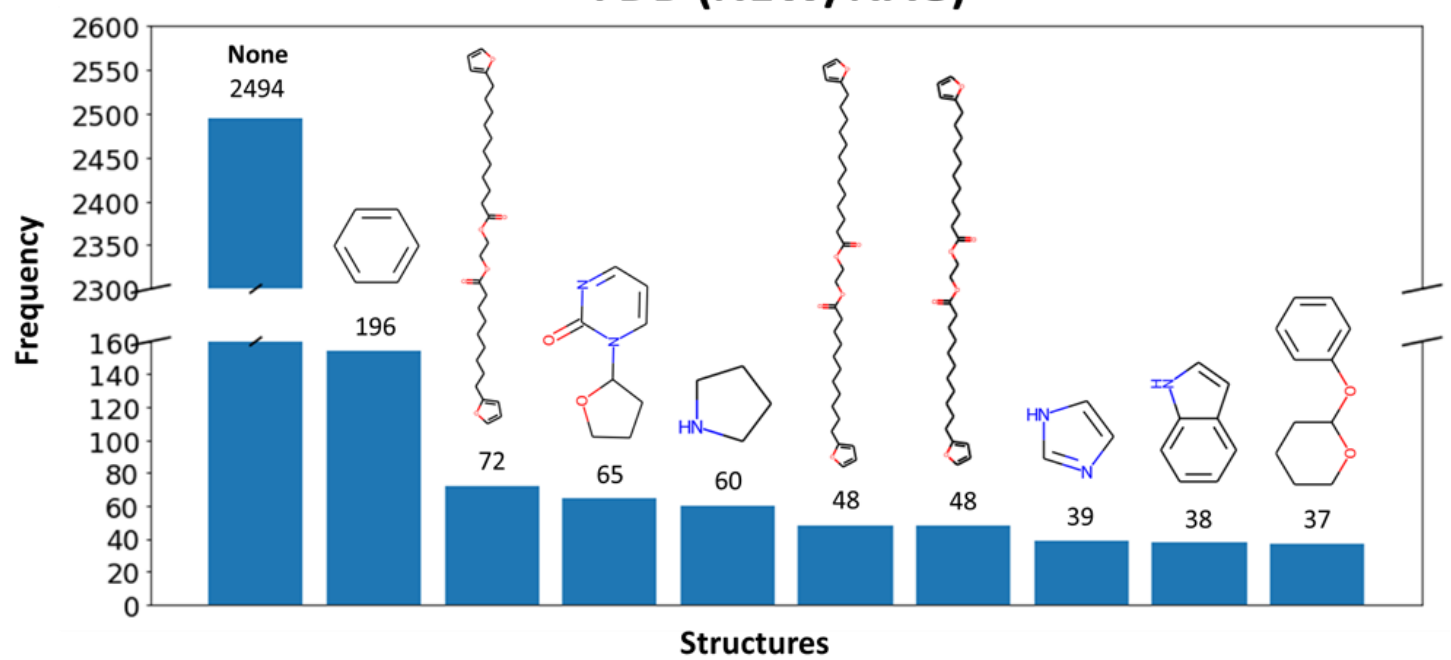

DB

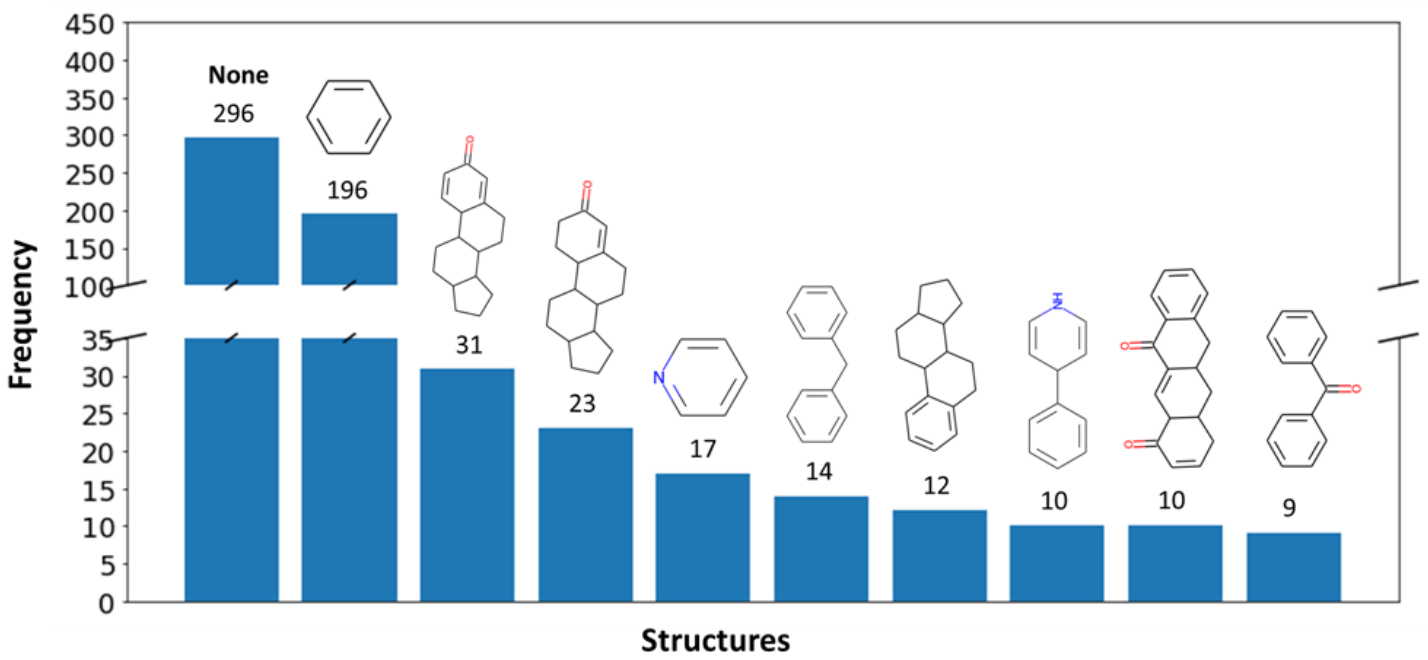

Figure 2. 
$\beta$-Lactamase

号

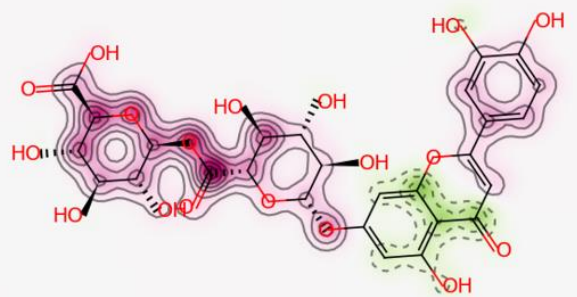

Non-Aggregator

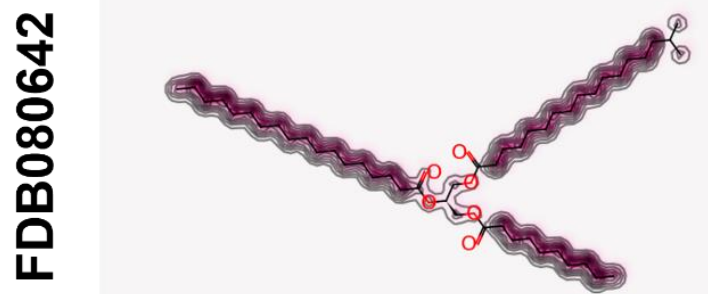

Aggregator
Cruzain

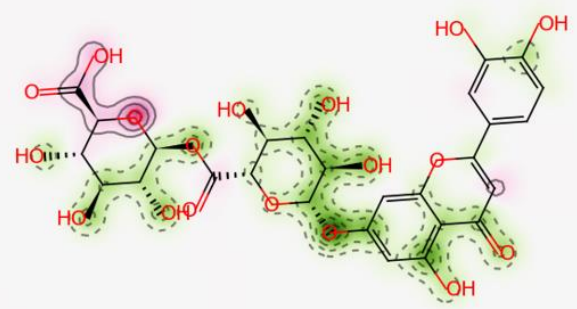

Non-Aggregator

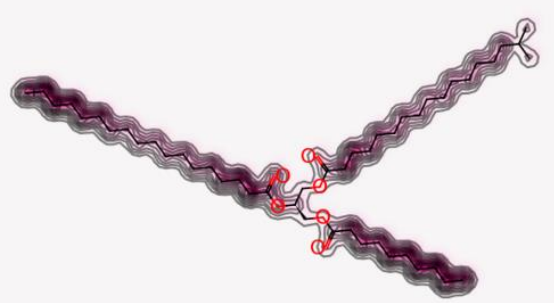

Aggregator

Figure 3. 


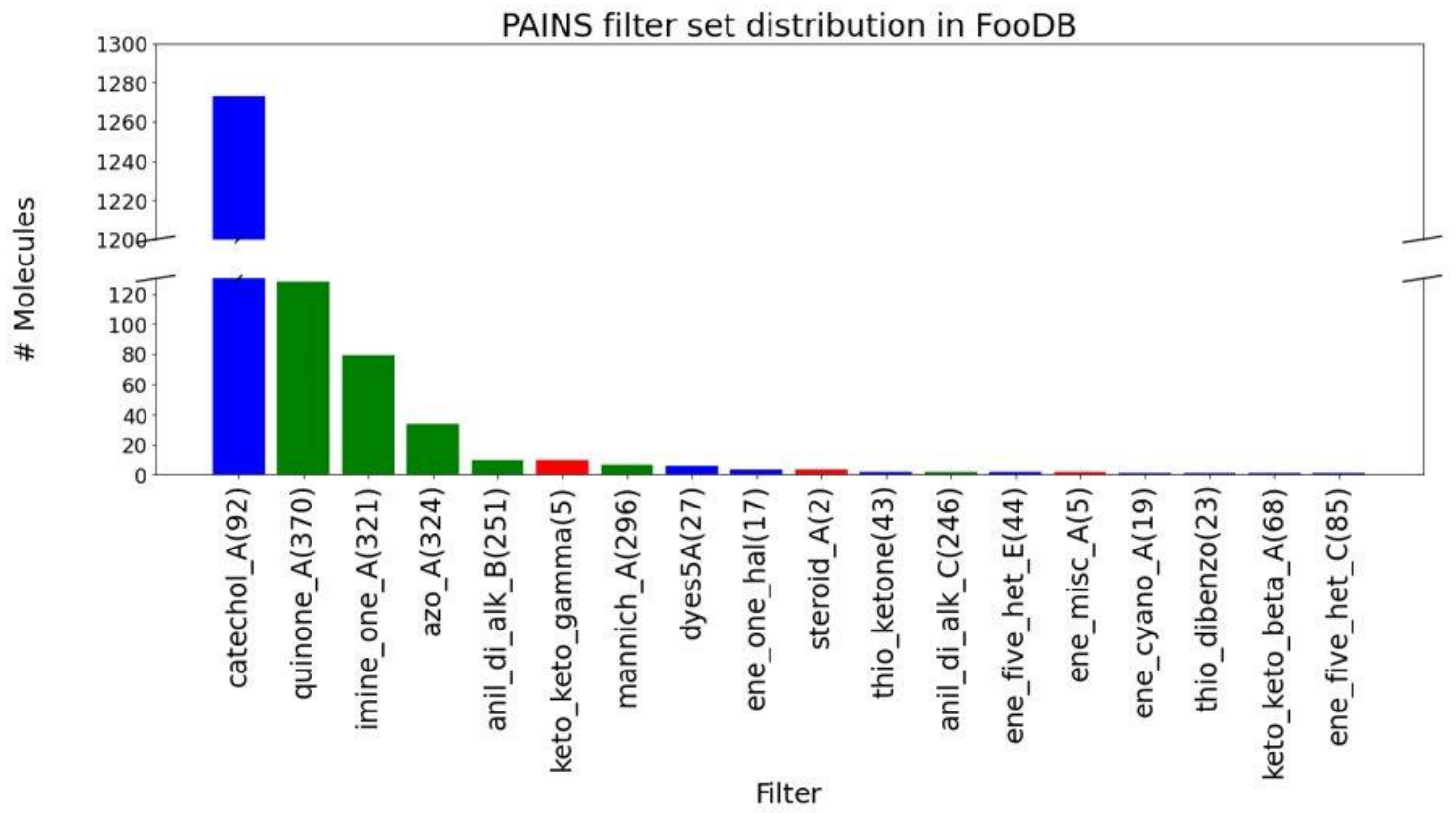

Figure 4. 


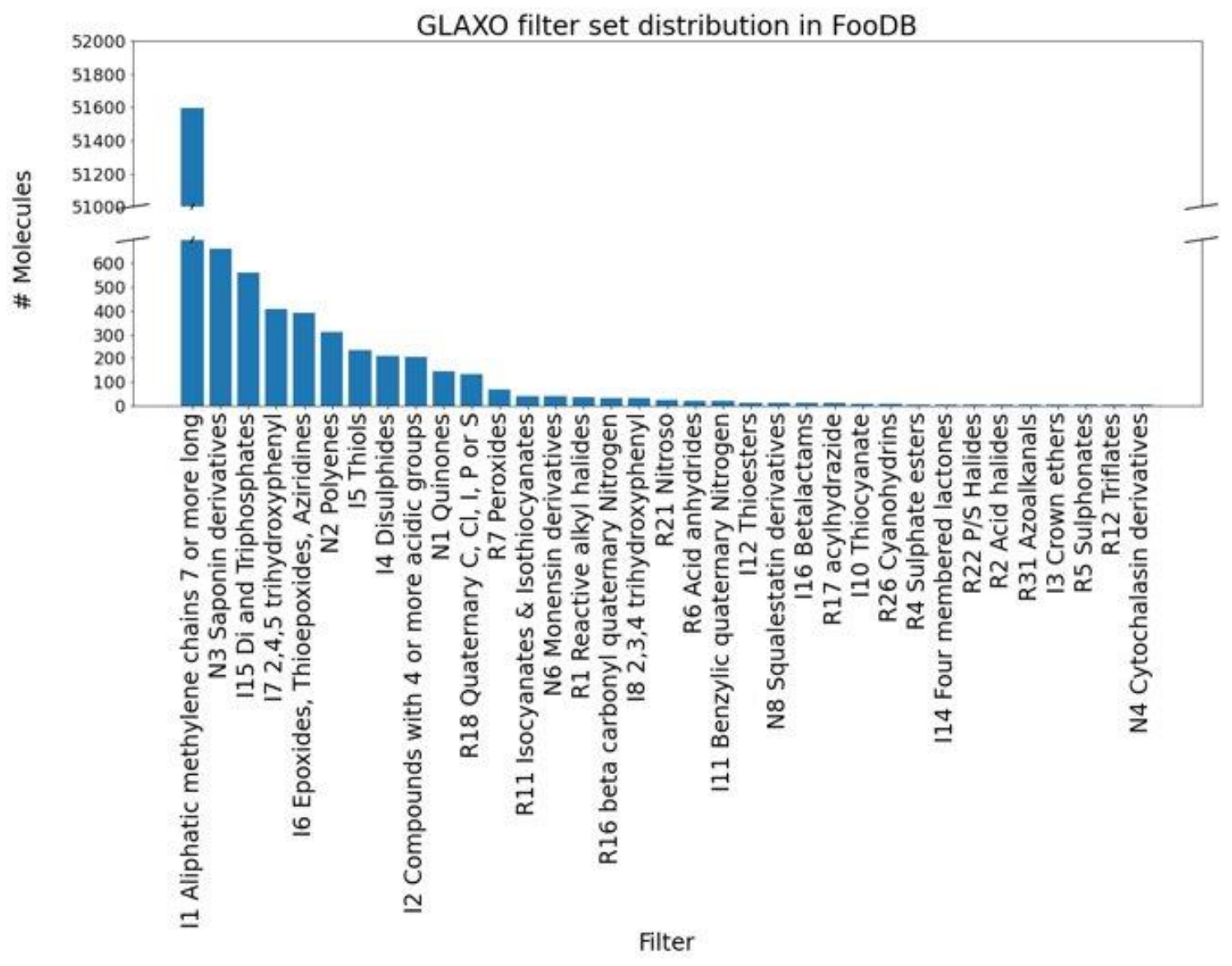

Figure 5. 


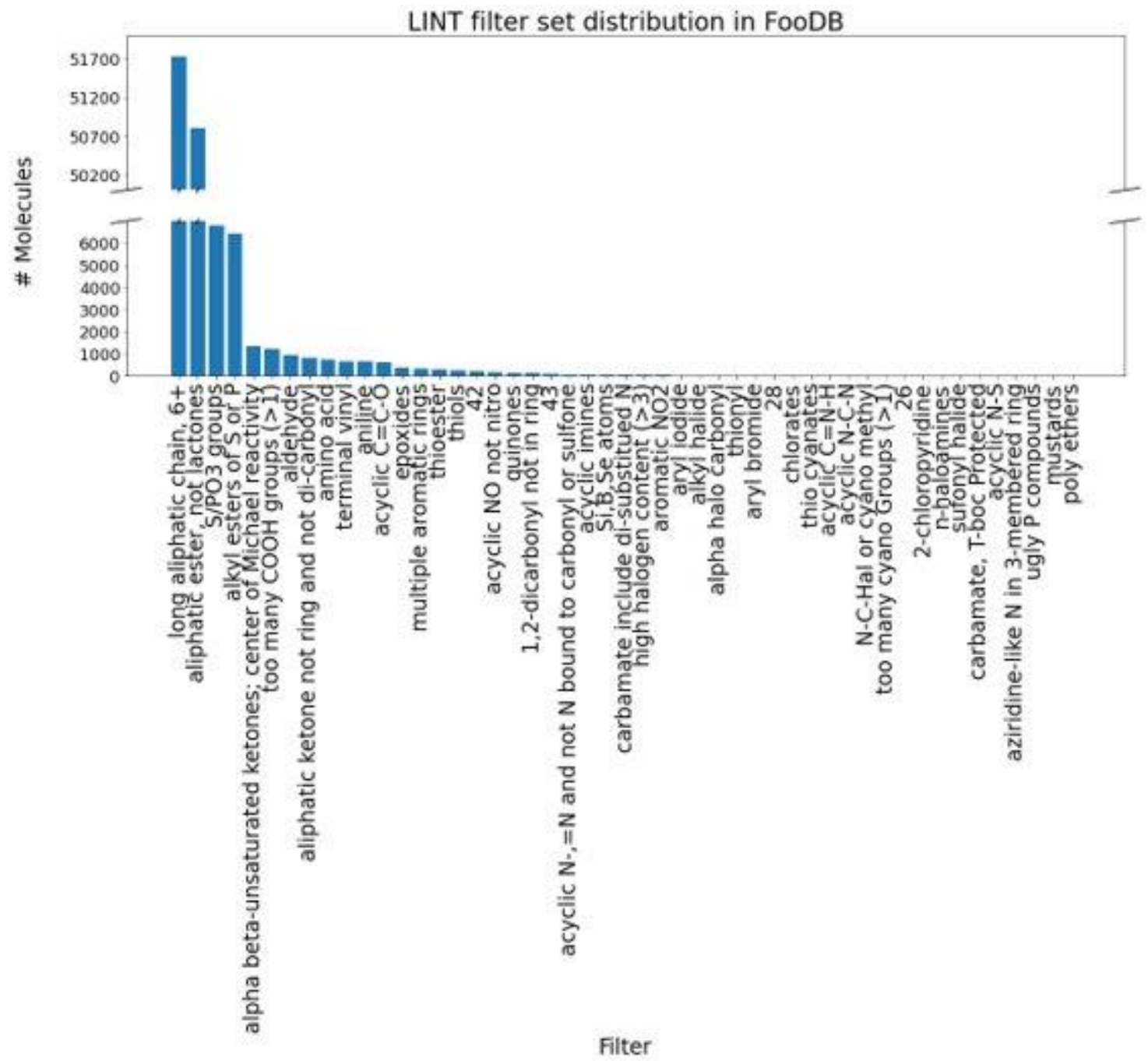

755 
Figure 6.TABLE OF CONTENTS GRAPHIC

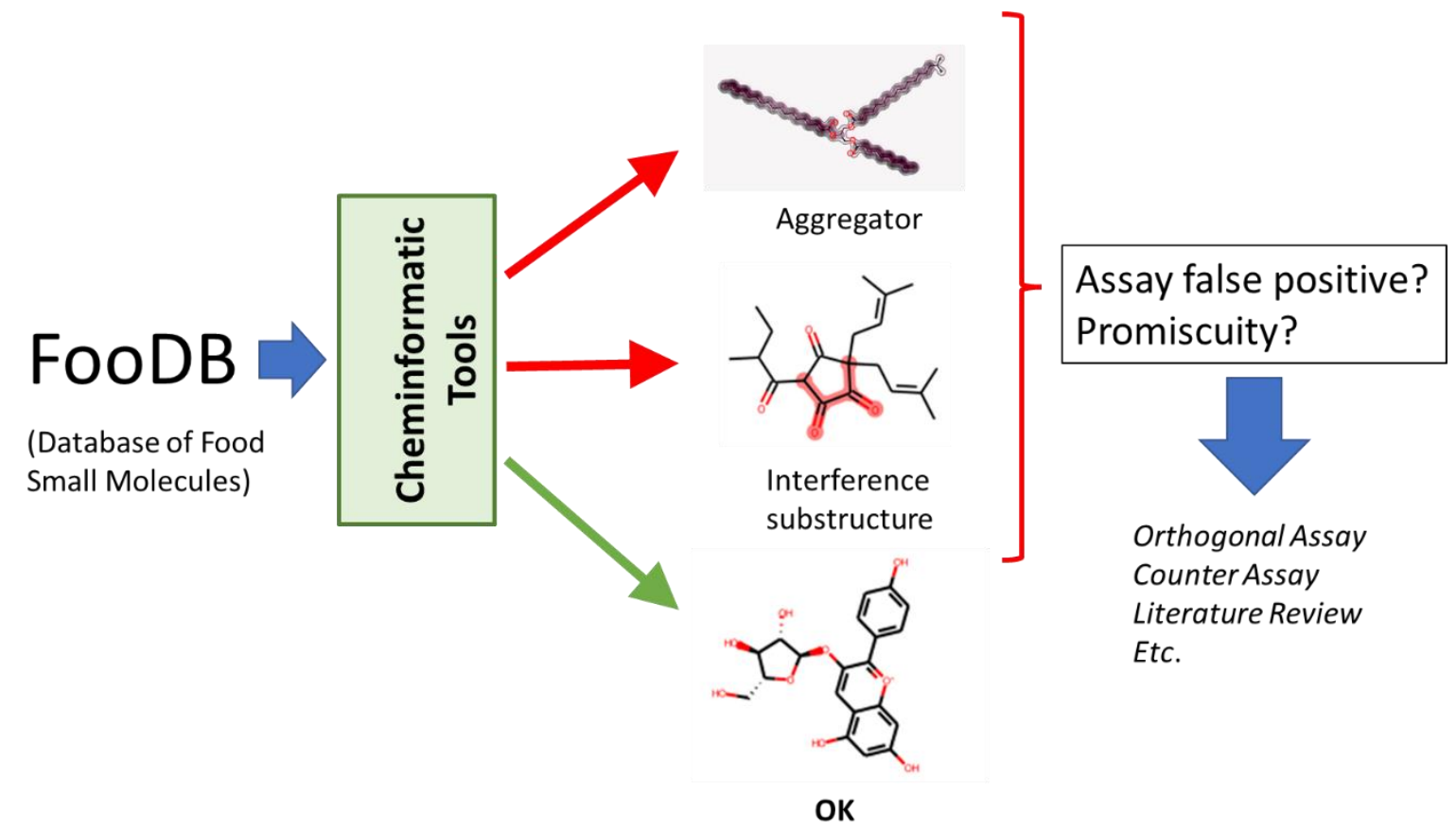

